\title{
Comparison of Antibody Responses Induced by RV144, VAX003, and VAX004 Vaccination Regimens
}

\author{
Chitraporn Karnasuta, Siriwat Akapirat, Sirinan Madnote, Hathairat Savadsuk,, Jiraporn Puangkaew, \\ Surawach Rittiroongrad, Supachai Rerks-Ngarm, Sorachai Nitayaphan, ${ }^{3}$ Punnee Pitisuttithum, \\ Jaranit Kaewkungwal, James Tartaglia, Faruk Sinangil,? Donald P. Francis, Merlin L. Robb,,9 \\ Mark S. de Souza, ${ }^{10}$ Nelson L. Michael, Jean-Louis Excler, ${ }^{11}$ Jerome H. Kim, ${ }^{11}$ \\ Robert J. O'Connell, and Nicos Karasavvas ${ }^{1,12}$
}

\begin{abstract}
The RV144 prime-boost regimen demonstrated efficacy against HIV acquisition while VAX003 and VAX004 did not. Although these trials differed by risk groups, immunization regimens, and immunogens, antibody responses may have contributed to the differences observed in vaccine efficacy. We assessed HIV-specific IgG, both total and subclass, and IgA binding to HIV envelope (Env): gp120 proteins and Cyclic V2 (CycV2) and $\mathrm{CycV} 3$ peptides and gp70 V1 V2 scaffolds in these $3 \mathrm{HIV}$ vaccine trials. After two protein immunizations, IgG responses to 92TH023 gp120 (contained in ALVAC-HIV vaccine) were significantly higher in RV144 but responses to other Env were higher in the VAX trials lacking ALVAC-HIV. IgG responses declined significantly between vaccinations. All trials induced antibodies to gp70 V1 V2 but VAX004 responses to $92 \mathrm{TH} 023$ gp70 V1 V2 were weak. All CycV2 responses were undetectable in VAX004 while 92TH023 gp70 V1 V2 was detected in both RV144 and VAX003 but MN CycV2 was detected only in VAX003. Multiple protein vaccinations in VAX trials did not improve magnitude or durability of V1 V2 and CycV2 antibodies. Herpes simplex virus glycoprotein $\mathrm{D}(\mathrm{gD})$ peptide at the $\mathrm{N}$ terminus of AIDSVAX ${ }^{\circledR} \mathrm{B} / \mathrm{E}$ and $\mathrm{B} / \mathrm{B}$ gp120 proteins induced antibodies in all trials, although significantly higher in VAX trials. gD peptide induced $\operatorname{IgA}, \operatorname{IgG} 1$, $\mathrm{IgG} 2$, and IgG3 but not IgG4. Multiple protein vaccinations decreased IgG3 and increased IgG4 changing subclass contribution to total IgG. Although confounded by different modes of HIV transmission, higher Envspecific IgA and IgG4 binding antibodies induced in the VAX trials compared to RV144 raises the hypothesis that these differences may have contributed to different vaccine efficacy results.
\end{abstract}

Keywords: HIV vaccines, RV144, VAX003, VAX004, antibodies, gp120, V2, V3, V1 V2, IgG, IgG subclasses IgA, HSV

${ }^{1}$ Department of Retrovirology, Armed Forces Research Institute of Medical Sciences (AFRIMS), Bangkok, Thailand.

${ }^{2}$ Department of Disease Control, Ministry of Public Health, Nonthaburi, Thailand.

${ }^{3}$ Royal Thai Army, AFRIMS, Bangkok, Thailand.

${ }^{4}$ Vaccine Trial Centre, Faculty of Tropical Medicine, Mahidol University, Bangkok, Thailand.

${ }^{5}$ Center of Excellence for Biomedical and Public Health Informatics, Faculty of Tropical Medicine, Mahidol University, Bangkok, Thailand.

${ }^{6}$ Sanofi Pasteur, Swiftwater, Pennsylvania.

${ }^{7}$ Global Solutions for Infectious Diseases (GSID), South San Francisco, California.

${ }^{8}$ U.S. Military HIV Research Program, Walter Reed Army Institute of Research, Silver Spring, Maryland.

${ }^{9}$ Henry M. Jackson Foundation for the Advancement of Military Medicine, Bethesda, Maryland.

${ }^{10}$ The Thai Red Cross AIDS Research Centre, Bangkok, Thailand.

${ }^{11}$ International Vaccine Institute, Seoul, Republic of Korea.

${ }^{12}$ Viral Diseases Branch, Walter Reed Army Institute of Research, Silver Spring, Maryland.

(C) Chitraporn Karnasuta et al. 2017; Published by Mary Ann Liebert, Inc. This article is available under the Creative Commons License CC-BY-NC (http://creativecommons.org/licenses/by-nc/4.0). This license permits non-commercial use, distribution and reproduction in any medium, provided the original work is properly cited. Permission only needs to be obtained for commercial use and can be done via RightsLink. 


\section{Introduction}

$\mathbf{T}$ he Thai Phase III trial, RV144 (ClinicalTrials.gov NCT00223080), provided the first evidence that an HIV1 vaccine could confer protective efficacy against HIV-1 acquisition. ${ }^{1}$ The prime-boost vaccine regimen consisted of a nonreplicating recombinant canarypox vector, ALVAC-HIV prime (vCP1521) and AIDSVAX ${ }^{\circledR}$ gp120 B/E boost. The modified intent-to-treat analysis showed $31.2 \%$ efficacy after 42 months of follow-up after vaccination. In a post hoc analysis, vaccine efficacy was $60 \%$ at 12 months post initial vaccination, suggesting early, but nonsustained, vaccine protection. $^{2}$ In the analysis of the correlates of risk (CoR), two variables correlated significantly with HIV-1 infection risk: plasma IgG binding antibody to scaffolded variable loops 1 and 2 (V1 V2) proteins (gp70 V1 V2) correlated inversely with risk for HIV-1 acquisition while Env plasma IgA (monomeric) binding score correlated directly with risk, raising the hypothesis that IgA responses against Env and IgG responses directed against V1 V2 may be mechanistically associated with RV144 vaccine regimen-mediated protection. ${ }^{3-5}$ A viral sieve analysis supported the potential role of anti V2 vaccine-induced antibodies in the reduction of HIV infection risk in RV144. ${ }^{6}$

Combinatorial polyfunctionality analysis of antigenspecific $\mathrm{T}$ cell subsets (COMPASS) showed that vaccines induced polyfunctional $\mathrm{CD} 4^{+} \mathrm{T}$ Cell subsets that were associated with decreased risk of HIV infection in RV144. ${ }^{7}$ These subsets included CD40 L and IL4 that are important for CD4 ${ }^{+}$ $\mathrm{T}$ cell-B cell interactions. Therefore, $\mathrm{CD} 4^{+} \mathrm{T}$ cell subsets may have contributed to $\mathrm{T}$ cell help for antibody production detected as a correlate in primary analysis. In RV144, polyfunctional effector memory $\mathrm{CD}^{+} \mathrm{T}$ cells were directed to HIV-1 Env particularly to the V2 loop region. ${ }^{8}$

AIDSVAX B/E alone conferred no protection among Thai people who inject drugs (PWID) (VAX003, ClinicalTrials.gov NCT00006327). ${ }^{9}$ Similarly, no overall significant protection was seen with a similar regimen of bivalent gp120 (AIDSVAX B/B) alone (VAX004, ClinicalTrials.gov NCT00002441) among mostly men who had sex with men (MSM) in North America and The Netherlands. ${ }^{10}$ Vaccine regimens in all three clinical trials elicited Tier-1, typespecific neutralizing antibodies (NAb). ${ }^{11,12}$ The peak NAb response ( 2 weeks post fourth vaccination) in RV144 was weaker than in VAX003. Only Tier-1 viruses were neutralized in RV144 and mediated in part by antibodies against the gp120 V3 loop. ${ }^{12}$ Low neutralizing activity was occasionally detected against Tier-2 viruses in the more sensitive A3R5 $\mathrm{NAb}$ assay. Peak responses in both trials waned considerably 6 months postimmunization. ${ }^{12}$ In VAX004, strong NAb responses were seen against a subset of Tier-1 viruses with sporadic weak responses against Tier- 2 viruses. ${ }^{13}$

Peptide microarray analysis from six HIV-1 subtypes and group M consensus showed that the RV144 vaccination regimen induced antibody responses to the V2 loop of gp120 of multiple subtypes and recognized both conformational and linear epitopes. ELISA and surface plasmon resonance assays using cyclic (Cyc) and linear V2 loop peptides showed that a majority of samples tested $(97 \%)$ had antibody responses against Cyclic V2 (CycV2) 2 weeks post last protein injection. The frequency of V2 antibodies declined to $19 \%$ at 28 weeks post last vaccination. Antibody responses targeted the mid-region of the V2 loop that contains conserved epitopes. ${ }^{14}$ The frequency of binding antibodies to CycV3 92TH023 and A244 peptides was modest whereas gp120 MN induced strong V3-specific antibodies. ${ }^{14-17}$

A comparison of vaccine-induced immune responses using a custom multiplex antibody assay demonstrated significantly higher anti-gp70 V1 V2 IgG3 response rates in RV144 than in VAX003 vaccines, suggesting a class-switching effect perhaps induced by the ALVAC-HIV priming. ${ }^{15}$ In RV144, IgG3 was correlated with decreased risk of HIV-1 infection. Anti-Env IgG3 levels significantly correlated with Tier-1 neutralization and ADCC in RV144 but not in VAX003. ${ }^{15}$ Although these results suggest that the differences of IgG3 levels might at least partially underlie different efficacy outcomes in these two trials, a strict comparison of the two trials is difficult since the routes of transmission were fundamentally different (mostly heterosexual vs. PWID). Systems serology studies indicated that IgG3 may serve as a surrogate of vaccine induced IgG1 that directs the polyfunctional antibody response observed in RV144. ${ }^{18}$ Therefore, the generation of IgG3 in RV144 could have initiated a coordinated production of poly-functional IgG1 with enhanced Fc-receptor binding characteristics.

To better understand the antibody responses associated with vaccine efficacy, we assessed the specificity of the antibody responses to gp120 proteins, V1 V2 scaffolds, CycV2 and $\mathrm{CycV} 3$ peptides in RV144 samples and compared them with those induced by the monomeric gp120 proteins tested in VAX003 and VAX004 efficacy trials. We also investigated the IgG subclasses of the antibody responses to V1 V2 and Env proteins.

\section{Materials and Methods}

\section{Vaccines and immunization regimens}

ALVAC-HIV (vCP1521) (Sanofi Pasteur) is a recombinant canarypox vector genetically engineered to express gp120 of HIV-1 subtype CRF01_AE 92TH023 strain linked to the transmembrane anchoring portion of subtype B gp41 (with a deletion in the immunodominant region devoid of the entire gp41 ectodomain), and HIV-1 gag and protease (both LAI strain). ALVAC-HIV (vCP1521) was formulated at a dose of $10^{6.5}$ CCID $_{50 .}{ }^{1}$ AIDSVAX B/E [Global Solutions for Infectious Diseases (GSID)], a bivalent vaccine, consisted of recombinant gp120 proteins (rgp120) from HIV-1 subtype B MN strain and HIV-1 subtype CRF01_AE A244 (CM244) strain both produced in Chinese hamster ovary cells, adsorbed onto alum and administered at $600 \mu \mathrm{g}$ doses (300 $\mu \mathrm{g}$ of each rgp120). ${ }^{1,9}$ AIDSVAX B/B (GSID), also a bivalent vaccine, consisted of $300 \mu \mathrm{g}$ each of MN and HIV-1 subtype B GNE8 proteins adsorbed onto alum. Unlike $\mathrm{MN}$ and CM244, GNE8 gp120 was cloned directly from human peripheral-blood mononuclear cells and has the CCR5 phenotype. $^{19}$

RV144 participants were vaccinated intramuscularly with ALVAC-HIV at weeks 0, 4, 12, and 24, with coadministration (in separate intramuscular injections) of AIDSVAX B/E rgp120 at weeks 12 and 24. VAX003 and VAX004 participants received AIDSVAX B/E and AIDSVAX B/B, respectively, intramuscularly at weeks $0,4,24,52$, 72,104 , and 144 in the absence of ALVAC-HIV priming (Fig. 1A). 


\section{Plasma and serological specimens}

All three completed clinical trials were approved by the appropriate Institutional Review Boards, Ethics Committees, and Regulatory Authorities. Written informed consent was obtained from all volunteers.

RV144 volunteer plasma samples were randomly selected by the Statistical Center for HIV/AIDS Research Prevention (SCHARP), and the EMMES Corporation, from volunteers who had received all immunizations per protocol and remained HIV-uninfected at the end of the study (with a 4:1 vaccinee:placebo ratio and for the availability of the biological specimens). RV144 plasma samples were obtained from 32 vaccine and 8 placebo recipients (Set Z) and assayed at baseline, 2 weeks post last injection (visit 8) and 6 months after completion of all vaccinations (visit 9) (Fig. 1A). Sera from VAX003 and VAX004 were provided by GSID and were obtained from 50 and 30 vaccine and 20 and 10 placebo recipients, respectively. Samples were analyzed at baseline (A02) and at subsequent visits (A04-A13) (Fig. 1A).

\section{Recombinant proteins and peptides}

rgp120 HIV-1 CRF01_AE A244gD, 92TH023 and subtype B MNgD proteins (identical to that used in RV144) were expressed in 293T cells and purified on Galanthous nivalis lectin columns. ${ }^{14}$ The rgp120 glycoprotein D (gD) proteins contained 27 amino acids of the herpes simplex virus 1 (HSV1) antigen and 3 amino acid linkers at the $\mathrm{N}$ terminus. ${ }^{19,20}$ Recombinant fusion gp70 V1 V2 glycoproteins containing the variable loops of V1 and V2 of gp120 were described previously. ${ }^{21}$ Subtype B Case A2 ${ }^{22}$ and CRF01_AE 92 TH023 gp70 V1 V2 scaffolds were synthetized as previously described and provided by Dr. Abraham Pinter. ${ }^{21,22}$

Cyclic (Cyc) peptides (JPT Peptide Technologies) were cyclized by disulfide bond formation with a purity $>90 \%$ measured by high-pressure liquid chromatography and mass spectrometry. Amino acid sequences of $\mathrm{CycV} 2$ and $\mathrm{CycV} 3$ peptides were based on the Env glycoproteins of $92 \mathrm{TH} 023$ (accession number EF553537.1, GenBank) and MN strains (Fig. 1B, C). A244 and 92TH023 proteins have identical V2 sequences at the antibody binding regions ${ }^{14}$ and their V3 loop regions vary by one amino acid at position 308 (Fig. 1B). HSV-1 (gD peptide) and HSV-2 peptides have the following sequences, KYALADASLKMADPNRFRGKDLPVLDQ and KYALADPSLKMADPNRFRGKNLPVLDQ, respectively (amino acids that differ from the HSV-1 sequence are underlined), purity $>90 \%$ (AAPPTEC).

\section{ELISA with cyclic peptides and recombinant proteins}

All samples were subjected to ELISA screening and titration (initial dilution 1:100 unless indicated) was performed on samples showing absorbance greater than 0.25 (2.5 times the background of wells without capturing antigen) at absorbance of $405 \mathrm{nM}$. ELISA was performed as described previously. ${ }^{14}$ For IgG subclasses (IgG1-IgG4) plates were coated with antigen as in regular ELISA and plasma was initially diluted 1:25 in blocking buffer and then serial twofold dilutions were performed and added to wells for 1-h incubation at room temperature. Wells were washed and mouse anti-human IgG1 or IgG3 (Invitrogen), or mouse antihuman IgG2 or IgG4 (Southern Biotech) were added for an hour at room temperature. Plates were washed and horseradish peroxidase (HRP) conjugated goat anti-mouse IgG (Southern Biotech) was added and incubated for $1 \mathrm{~h}$ at room temperature. Plates were washed, ABTS ELISA HRP substrate (KPL) was added, and color was allowed to develop at room temperature for $1 \mathrm{~h}$ in the dark. Plates were read at A405 nM using ELISA reader Spectramax 340 PC (Molecular Devices). In absorption studies, plasma/serum was preincubated with 10 micrograms of peptide for $1 \mathrm{~h}$ at room temperature before adding to ELISA plates (Fig. 3A, B).

\section{Statistical methods}

ELISA antibody titers were calculated using serial twofold dilutions of plasma/serum starting from 1:100 or $1: 25$ for IgG subclasses and expressed as the reciprocal of the highest dilution that yielded an absorbance value above 2.5 times the background value (wells without capturing antigen). An overall false positive response for each protein and peptide and by clinical trial was calculated based on 95th percentile from all baseline absorbance data of vaccine recipients. Antibody responses to an individual protein or peptide were expressed as percentage of subjects with a positive response, defined as A405 value $>0.25$ (positive response rate). Magnitude of antibody responses (including values below cutoff) to an individual protein or peptide was expressed as geometric mean titer (GMT) with 95\% confidence interval. Data analyses and graphs were generated using Graphpad Prism version 5 (GraphPad Software). Statistical comparisons of optical density (absorbance at $405 \mathrm{nM}$ ) for screening, and of specific antibody titers against rgp120 proteins, cyclic peptides, and V1 V2 scaffolds were made using nonparametric tests and a two-sided $p$ value of $<.05$ was considered significant. Multiple hypotheses performed were adjusted by controlling the false discovery rate at the 0.05 level.

\section{Results}

RV144 participants were intramuscularly vaccinated with ALVAC-HIV (vCP1521, a recombinant canarypox vector expressing gp120 of HIV-1 subtype CRF01_AE 92 TH023 strain linked to the trans-membrane portion of subtype B gp41) at weeks $0,4,12$, and 24 (visits 1, 3, 5, and 7, respectively), with co-administration (in separate intramuscular injections) of AIDSVAX B/E rgp120 from HIV-1 subtype B MN strain and HIV-1 subtype CRF01_AE A244 (CM244) at weeks 12 and 24 (visits 5 and 7, respectively, Fig. 1A). We analyzed RV144 antibody responses at baseline (week 0, visit 1), weeks 26 (2 weeks post last vaccination, visit 8) and 52 (6 months post last boost, visit 9). VAX003 participants received AIDSVAX B/E (as in RV144) and VAX004 participants received AIDSVAX B/B (a bivalent vaccine containing HIV-1 subtype B MN and GNE8 proteins) intramuscularly at weeks 0, 4, 24, 52, 72, 104, and 144 (visits A02, A04, A06, A08, A10, A12, and A14, respectively) in the absence of ALVAC-HIV priming (Fig. 1A, vaccinations occurred at even visits and odd visits represent 2 weeks postimmunization). Amino acid sequence alignment of variable loops two and three are shown in Figure 1B and percent identity and divergence of recombinant proteins used in all vaccines, with $\mathrm{HXB} 2$ used as a reference stain, are shown in Figure 1C. 
A

\begin{tabular}{|c|c|c|c|c|c|c|c|c|c|c|c|c|c|c|}
\hline & Weeks & 0 & 2 & 4 & 6 & 12 & 24 & 26 & 52 & 54 & 72 & 74 & 104 & 106 \\
\hline & RV144 Visits & V1 & & V3 & & V5 & V7 & V8 & V9 & & & & & \\
\hline \multirow{4}{*}{ Vaccinations } & RV144 & A & & A & & $\mathrm{A}+\mathrm{B} / \mathrm{E}$ & $\mathrm{A}+\mathrm{B} / \mathrm{E}$ & & & & & & & \\
\hline & VAX003 & $\mathrm{B} / \mathrm{E}$ & & $\mathrm{B} / \mathrm{E}$ & & & $\mathrm{B} / \mathrm{E}$ & & $\mathrm{B} / \mathrm{E}$ & & $\mathrm{B} / \mathrm{E}$ & & $\mathrm{B} / \mathrm{E}$ & \\
\hline & VAX004 & $\mathrm{B} / \mathrm{B}$ & & $\mathrm{B} / \mathrm{B}$ & & & $\mathrm{B} / \mathrm{B}$ & & $\mathrm{B} / \mathrm{B}$ & & $\mathrm{B} / \mathrm{B}$ & & $\mathrm{B} / \mathrm{B}$ & \\
\hline & VAX003/4 Visits & A02 & $\mathrm{A} 03$ & A04 & A05 & & A06 & A07 & A08 & A09 & A10 & A11 & A12 & A13 \\
\hline
\end{tabular}

B
HxB2_V2
$157 \quad 160$
170
180
190
196
CSFNI TTSI GDKMAKE YALLYKL DI EPI DNDSTS - YRLIS
GNE8_V2 CSFNVTTSI RDKMKNEYALFYKLDVVPI DNDNTS. - YRL I S
92TH023_V2 C SF NMTTELRDKKAKVHALF YKL DI VPI EDNTSSSEYRL I NC
A244_V2 CSF NMTTELRDKKQKVHALF YKL DI VPI EDNNDSSEYRL NC
$\begin{array}{lllll} & 296 & 300 & 310 & 320\end{array}$
MN_V3 CTRPNYNKRKRI HI - GPGRAFYTTKNI KGTI RQAHC
GNE8_V3 CTRPNNNTRRSI HI. - GPGRAFYATGEI I GDI RQAHC
92TH023_V3 CTRPSNNTRTSI NI. - GP GQVFYRTGDI I GDI RKAYC
A244_V3 CTRPSNNTRTSITI. - GPGQVFYRTGDI I GDIRKAYC
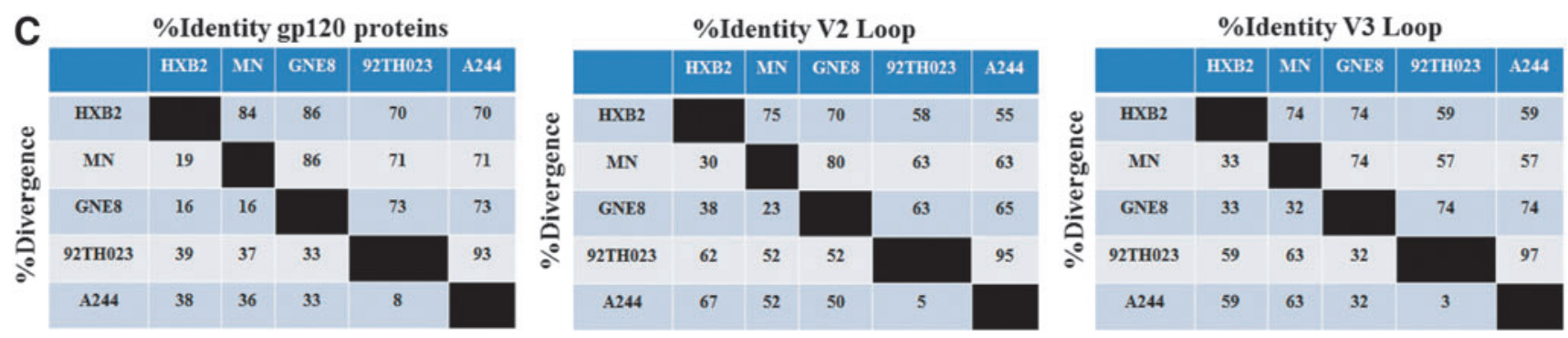

FIG. 1. RV144, VAX003, and VAX004 vaccination schedules and HIV-1 gp120 envelope variable loops 2 and 3 (V2 and V3). (A) RV144, VAX003 and VAX004 vaccination schedules and vaccine antigens; A = ALVAC-HIV (vCP1521), $\mathrm{B} / \mathrm{B}=\operatorname{AIDSVAX}^{\circledR} \mathrm{B} / \mathrm{B}(\mathrm{gp} 120 \mathrm{MNgD}$ and gp120 GNE8gD-600 $\mu \mathrm{g}), \mathrm{B} / \mathrm{E}=\mathrm{AIDSVAX} \mathrm{B} / \mathrm{E}(\mathrm{gp} 120 \mathrm{MNgD}$ and gp120 A244gD-600 $\mu \mathrm{g}$ ). Visit samples used in study are in bold. In VAX trials immunizations are given at even numbers and odd numbers represent 2 weeks post immunization. (B) Alignments of HIV-1 gp120 envelope variable loops 2 and 3 (V2 and V3); V2 and V3 loops are shown in the top and bottom panel, respectively. Boxed amino acids show differences from reference strain HxB2. Numbers on top of each panel are positions of amino acids based on the HxB2 strain. (C) Percent identity and divergence of gp120 proteins and variable loops 2 and 3 (V2 and V3). gD, glycoprotein D.

\section{Antibody responses to rgp120 Env proteins in RV144 and VAX trials}

RV144, VAX003, and VAX004 samples were tested for antibodies to $92 \mathrm{TH} 023, \mathrm{~A} 244 \mathrm{gD}$, and $\mathrm{MNgD}$ proteins at baseline. All three proteins are vaccine-matched to RV144 and $\mathrm{A} 244 \mathrm{gD}$ and $\mathrm{MNgD}$ are matched to VAX003 but only $\mathrm{MNgD}$ is matched to VAX004. Responses to gp120 GNE8 were not tested. No responses were detected against gp120 $92 \mathrm{TH} 023$ in any of the vaccinated individuals but at prevaccine baseline responses against $\mathrm{A} 244 \mathrm{gD}$ and $\mathrm{MNgD}$ were detected in RV144 (3/32, 9\%), VAX003 (6/50, 12\%) and VAX004 (7/30, 23\%) (data not shown).

We investigated the antibody binding to rgp120 proteins used in RV144, MNgD, and A244gD (contained in AIDS$\mathrm{VAX} \mathrm{B} / \mathrm{E}$ ) and 92TH023 (contained in ALVAC-HIV), at peak immunogenicity (week 26, visit 8), and compared them to those of VAX003 (AIDSVAX B/E) and VAX004 (AIDSVAX B/B) 2 weeks post second and fourth protein injections (weeks 6 and 54), A05 and A09, respectively (Fig. 2A-C). Protein vaccinations in the VAX trials and RV144 did not follow the same schedule. In RV144, the two protein inoculations were co-administered with ALVAC-
HIV at weeks 12 and 24, whereas in the VAX studies the first two protein inoculations occurred at weeks 0 and 4 and subsequent injections followed at 6-month intervals (Fig. 1A). VAX003 antibody responses to A244gD were significantly higher than in RV144 and VAX004 after two and four protein injections (Fig. 2A). Two weeks post second protein administration (week 6, A05), VAX003 GMT to $\mathrm{A} 244 \mathrm{gD}$ was 21,378 but in RV144 was 13,367 and in VAX004 was 14,084 (Fig. 2A). Two weeks post fourth protein administration (week 54, A09), VAX003 GMT increased to 42,757 but in VAX004 there was a slight decline $(10,159)$. Antibody responses to $\mathrm{MNgD}$ after two protein inoculations were similar in VAX003 $(24,900)$ and VAX004 $(25,600)$, statistically higher than those in RV144 $(15,896)$ (Fig. 2B). Two additional protein injections in VAX003 increase the GMT to 51,200 but remained steady in VAX004 (25,015). RV144 had significantly higher antibody responses to recombinant $92 \mathrm{TH} 023(6,263)$ compared to VAX003 $(3,430)$ and VAX004 $(1,639)$ after two protein administrations (Fig. 2C). GMT increased after two additional protein administrations in both VAX003 (24,557) and VAX004 $(6,550)$ but only VAX003 responses were statistically higher than RV144. 

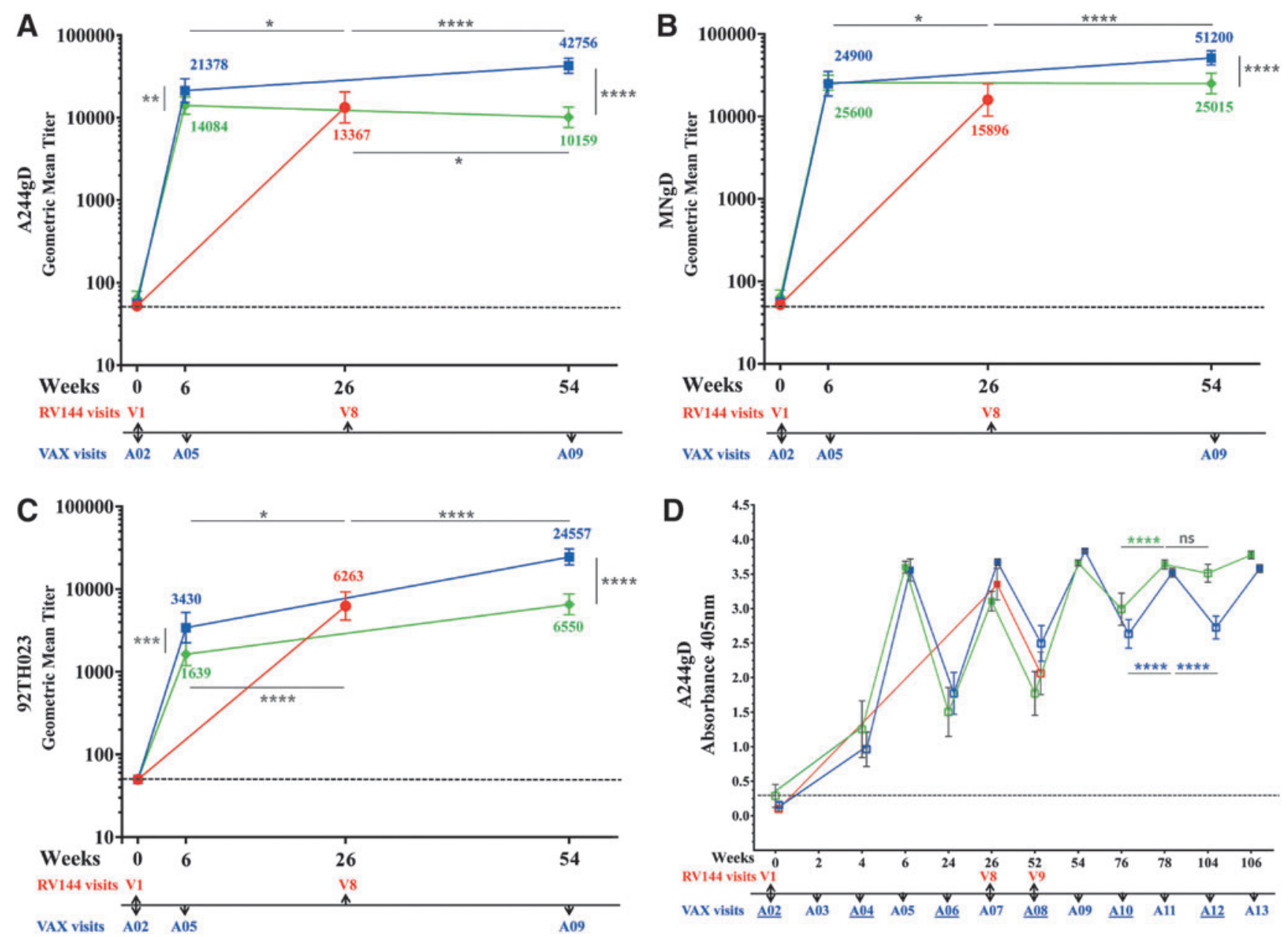

FIG. 2. IgG antibody binding to rgp120 envelope protein CRF01_AE A244gD, MNgD, and $92 \mathrm{TH} 023$ in RV144 and VAX trials. RV144 (red symbols, visits 1 and 8), VAX003 (blue symbols, visits A02, A05, and A09) and VAX004 (green symbols, visits A02, A05, and A09); symbols show GMTs of (A) A244gD, (B) MNgD, (C) 92TH023 with 95\% confidence intervals and color coded numbers depict actual GMT and (D) RV144 (red symbols, visits 1, 8, and 9), VAX003 (blue symbols, visits A02A13), and VAX004 (green symbols, visits A02-A13); solid symbols show 2 weeks post vaccination and hollow symbols show vaccination visits. Symbols show means of A244gD and bars are standard deviations at A405nm.VAX visit numbers are indicated below the $x$-axis with vaccination visits underlined. Responses above dotted line are considered positive. $* p<.05$; $* * p<.01 ; * * * p<.001 ; * * * * p<.0001$; ns, not significant. GMT, geometric mean titer; rgp120, recombinant gp120 proteins.

\section{Durability of antibody responses after multiple protein vaccinations in the VAX trials}

In RV144, the decline of vaccine efficacy $(60 \%$ at 12 months and $31.2 \%$ at 42 months) suggests an association with a parallel decline of antibody responses. ${ }^{2} \mathrm{We}$ investigated the persistence of antibody responses in VAX003 and VAX004 at peak immunogenicity and between vaccinations using the gp120 protein A244gD (Fig. 2D). In VAX003, maximum antibody responses were achieved 2 weeks post fourth protein injection (week 54, A09) and additional immunizations did not increase the magnitude of the response. Responses declined significantly between vaccinations and multiple immunizations did not maintain antibody titers at levels detected 2 weeks post boosting (Fig. 2D). In VAX004, multiple protein injections progressively increased the magnitude of antibody responses between vaccinations and antibody levels 2 weeks post fifth immunization (week 74, A11) were similar to those 6 months later (week 104, A12, Fig. 2D). Since VAX003 and VAX004 both included MNgD, the improved antibody persistence between vaccinations could be attributed to GNE8 gp120 protein.

\section{AIDSVAX gp120 gD-specific antibodies}

Recombinant AIDSVAX gp120 proteins, A244gD, $\mathrm{MNgD}$, and GNE8gD contained a 27-amino acid fragment from the HSV-1 gD at their $\mathrm{N}$ terminus to facilitate expression and immunoaffinity purification processes. ${ }^{19,20}$ The HSV-1 gD peptide shares a high homology with the HSV-2 gD: 25/27 amino acids are identical and differ at positions 32 (alanine to proline) and 46 (aspartic acid to asparagine) (see Materials and Methods section). Antibodies induced by the gD fragment of rgp120 could cross react with HSV-1 and 2 glycoproteins in natural infections. Similarly, antibodies induced by natural HSV infections could bind to the gD peptide on gp120. Using ELISA, we showed that gD-specific antibodies induce by the AIDSVAX gp120 proteins bind equally well to both HSV-1 and HSV-2 peptides (data not shown). 
At baseline, 3/32, 9.4\% (RV144), 6/50, 12\% (VAX003), and 7/30, 23.4\% (VAX004) volunteers had detectable antibody responses to $\mathrm{A} 244 \mathrm{gD}$ and $\mathrm{MNgD}$ gp120 s, but not to 92TH023, which does not contain the gD fragment. It was likely that these responses were targeting the $\mathrm{gD}$ and not the HIV-1 envelope sequences suggesting that some of the vaccinees were pre-exposed to HSV and had detectable circulating HSV antibodies ${ }^{23,24}$ before the AIDSVAX protein vaccinations. HSV-2 has been associated with increasing the risk of HIV-1 acquisition several fold. ${ }^{25-27}$ To test whether responses detected at baseline were gD-related, we preincubated baseline gp120 reactive samples with the gD peptide (identical sequence found in AIDSVAX proteins) to absorb preexisting binding antibodies before ELISAs using $\mathrm{A} 244 \mathrm{gD}$ as the capture antigen. In RV144, antibodies were not absorbed (0/3) but 5/6 samples in VAX003 and 6/7 in VAX004 were completely absorbed (data not shown) indicating the presence of circulating HSV antibodies in VAX vaccinees. We examined gD-specific antibody responses induced by gp120 Env proteins by absorbing gD responses in RV144, VAX003, and VAX004 2 weeks post second protein vaccination (visits 8 , week 26 and $A 05$, week 6 , respectively) using A244gD. Absorption of $\mathrm{IgG}$ antibodies to the $\mathrm{gD}$ peptide in RV144 decreased the GMT from 13,367 to 11,995 (not significant, $p=.49$ ) but a significant reduction of antibody titers was seen in VAX003 and VAX004, declined from 21,378 to $11,716(p=.0054)$, and from 12,498 to 6,713 $(p=.0003)$, respectively (Fig. 3A). To determine the progression of $\mathrm{gD}$-specific antibodies between vaccinations we absorbed VAX004 sera with the gD peptide to remove $\mathrm{gD}$ specific antibodies. A significant drop in antibody binding was detected at all visits tested when $\mathrm{gD}$-specific antibodies were absorbed (Fig. 3B). However, the contribution of the $\mathrm{gD}$-specific $\mathrm{IgG}$ antibodies decreased with multiple protein administrations.

\section{Antibody responses to gp70 V1 V2 scaffolds}

We compared the generation V1 V2 antibodies in all three regimens. Antibody binding to gp70 V1 V2 scaffolds $92 \mathrm{TH} 023$ and subtype B Case A2 were not detected at baseline (Fig. 4A, B). Antibody responses to $92 \mathrm{TH} 023 \mathrm{gp} 70$ V1 V2 scaffold were detected in all three trials after two protein injections (Fig. 4A). GMT antibody responses to 92TH023 gp70 V1 V2 were highest in VAX003 $(2,904)$ and RV144 (2,559) and significantly higher than in VAX004 (65). In VAX003, two additional protein vaccinations increased the V1 V2 titers to 5,268 but responses in VAX004 remained low (79). Responses to gp70 V1 V2 subtype B Case A2 scaffold were significantly higher in RV144 (176) after two protein injections (visit 8, week 26) compared to visit A05 (week 6) in VAX003 (109) and VAX004 (70) (Fig. 4B). Two additional protein boosts (visit A09, week 54) significantly increased the response in VAX003 $(2,786)$ and VAX004 (553) that were significantly higher than those of RV144 (Fig. 4B).

To examine whether multiple protein boosts in VAX trials increase the antibody responses to V1 V2 scaffolds, we measured absorbance at 2 weeks and 6 months post protein vaccinations (Fig. 4C, D). In VAX004, responses to the $92 \mathrm{TH} 023$ scaffold were weak and close to baseline at visits A05 (week 6) and A09 (week 54) and below baseline at other
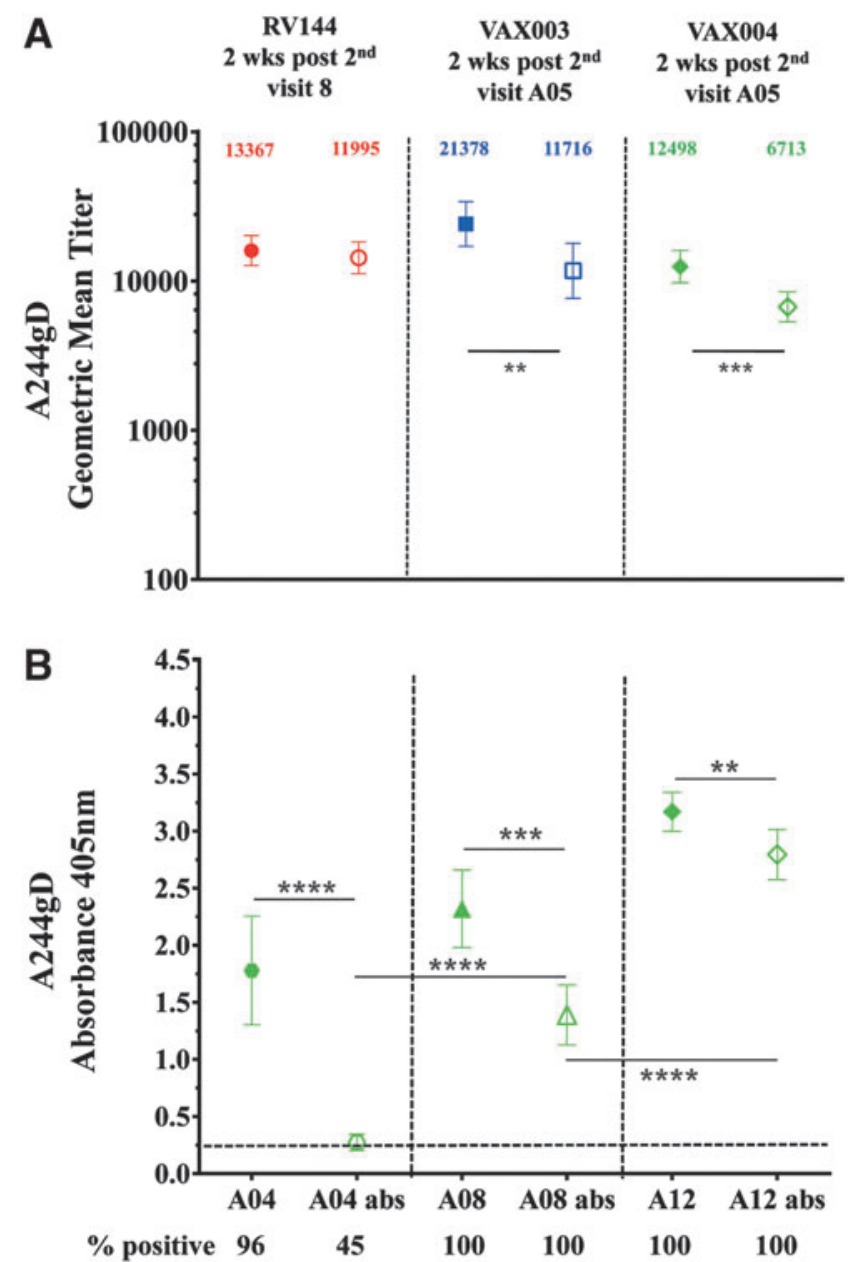

FIG. 3. IgG antibodies to the gD peptide from RV144 and VAX samples. RV144 (red symbols), VAX003 (blue symbols), and VAX004 (green symbols) at 2 weeks post second AIDSVAX vaccination from visits 8 and A05, respectively. (A) Symbols are GMTs showing absorption of IgG antibodies binding to the gD peptide with $95 \%$ confidence intervals. Numbers indicate GMTs before (solid symbols) or after absorption (hollow symbols) of the gD binding antibodies. (B) IgG specificity to gD after multiple immunizations in VAX004 serum samples from second (visit A04), fourth (visit A08), and sixth (visit A12) AIDSVAX B/B vaccinations. Solid symbols show sera before absorption and hollow symbols show absorbed sera with the $\mathrm{gD}$ peptide. Recombinant gp120 CRF01_AE A244gD was the capturing antigen. The plots are means with standard deviations at absorbance $405 \mathrm{~nm}$. $x$-axis shows visits. Numbers under $x$-axis show percentage of positive samples (A $405 \geq 0.25$ ) and responses above dotted line are considered positive. $* * p<.01 ; * * * p<.001 ; * * * * p<.0001$.

visits (Fig. 4C). In VAX003, antibodies to V1 V2 $92 \mathrm{TH} 023$ peaked 2 weeks post fourth vaccination (A09) and were significantly higher than in RV144 (visit 8, week 26). Additional vaccinations did not increase antibody responses to V1 V2 and responses declined significantly with additional boosts (Fig. 4C). Antibody levels between vaccinations were short lived and declined to baseline levels in all vaccines indicating that multiple immunizations in the VAX trials did not increase the durability of antibody response to this scaffold. 

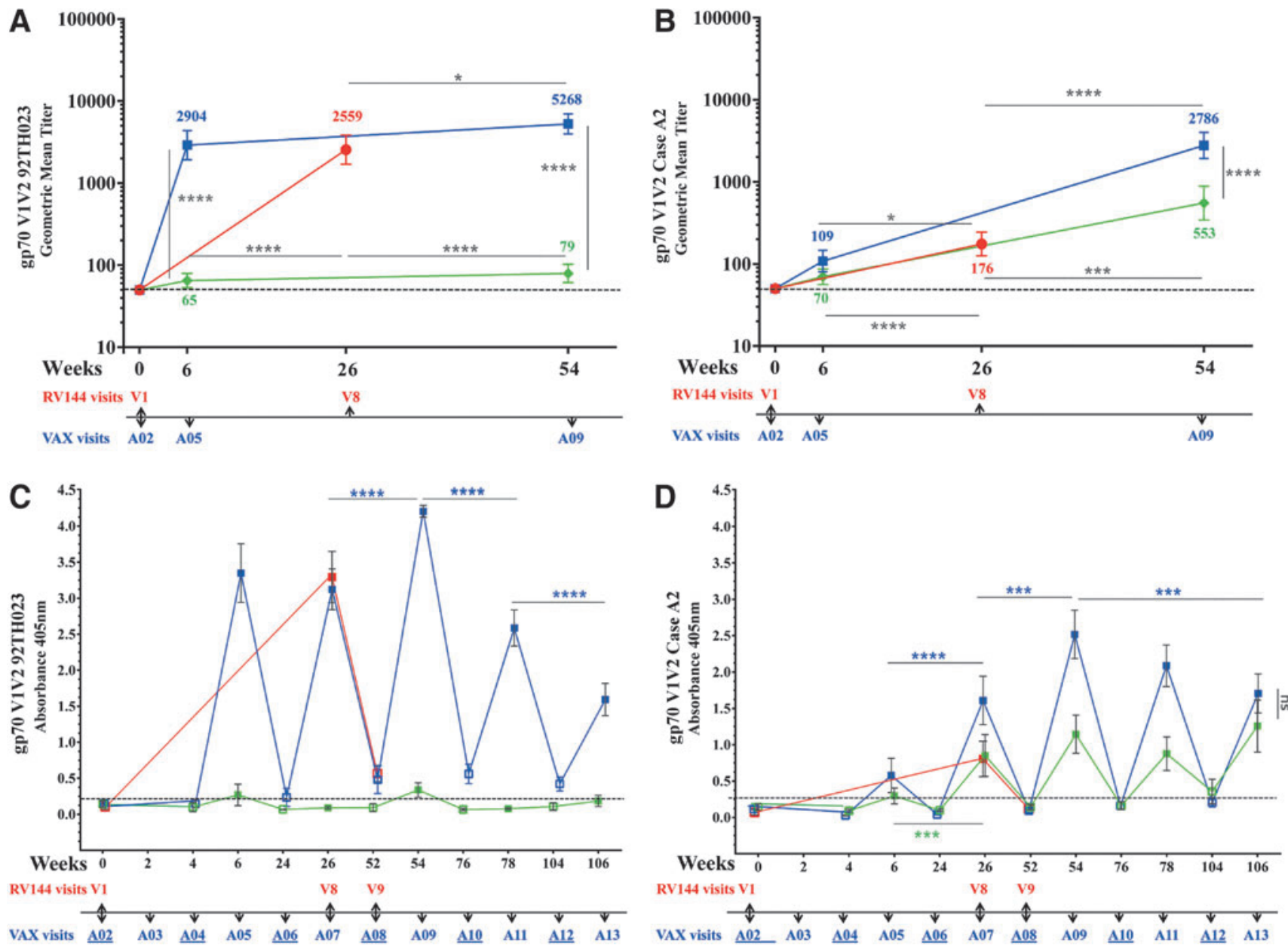

FIG. 4. IgG antibody binding to gp70 V1 V2 scaffold in RV144 and VAX trials. RV144 (red symbols), VAX003 (blue symbols), and VAX004 (green symbols); (A) and (B) symbols show GMTs of gp70 V1 V2 92TH023 and gp70 V1 V2 Case A2, respectively, with 95\% confidence intervals and color coded numbers depict actual GMTs of RV144 (visits 1 and 8), VAX003 (visits A02, A05, and A09), and VAX004 (visits A02, A05, and A09); (C) and (D) show means and bars are standard deviations of RV144 (visits 1, 8, and 9), VAX003 (visits A02-A13), and VAX004 (visits A02-A13) at A405nm of gp70 V1 V2 92TH023 and gp70 V1 V2 Case A2, respectively. Solid symbols show 2 weeks post vaccination and hollow symbols show vaccination visits. VAX visit numbers are indicated below the $x$-axis with vaccination visits underlined. Responses above dotted line are considered positive. $* p<.05 ; * * * p<.001$; ****p<.0001; ns, not significant.

Antibody responses to gp70 V1 V2 subtype B Case A2 scaffold followed a similar pattern to $92 \mathrm{TH} 023$ with antibody binding declining to baseline levels 6 months post vaccinations in all trials (Fig. 4D). In both VAX trials responses peaked 2 weeks post fourth immunization (A09) but a decrease in antibody binding was seen in VAX003 with subsequent protein boosts (Fig. 4D).

\section{V2-specific antibody responses in RV144 and VAX trials}

Antibodies to V2 linear epitopes ${ }^{28}$ inversely correlated with risk for HIV-1 acquisition. The V2 loop also contains many conserved features including the putative $\alpha 4 \beta 7$ integrin binding motif, ${ }^{29}$ and several anti-V2 monoclonal antibodies show broad neutralizing cross-reactivity (reviewed in O'Connell et $a{ }^{30} .^{30}$, suggesting that antibodies can target conserved epitopes in the V2 loop and that this Env region might be important for the generation of vaccine-induced protective antibodies. ${ }^{14} \mathrm{We}$ tested antibody responses to 92TH023 and MN CycV2 peptides using samples from all trials at baseline (visits 1 and A02, week 0), 2 weeks post second (visits 8, week 26 and A05 week 6) and 2 weeks postfourth protein vaccinations (week 54, A09) (Fig. 5A, B). At baseline no antibody responses were detected to CycV2 MN (all trials) but two samples from RV144 were above cutoff with CycV2 92TH023 and considered not specific (data not shown). VAX004 vaccinations did not induce $\mathrm{CycV} 2$ 92TH023 antibodies (Fig. 5A). RV144 and VAX003 induced similar antibody responses (972 and 1,100, respectively) to CycV2 92TH023 after two protein vaccinations (Fig. 5A) and two additional protein boosts in VAX003 did not increase further the magnitude of the antibody response (1,041). In all three regimens, responses to $\mathrm{CycV} 2 \mathrm{MN}$ were undetectable 2 weeks post second protein boost, except for one sample in RV144 and two in VAX003 being above cutoff. Two additional protein boosts induced weak antibody responses in VAX003 (76) but not in VAX004. 

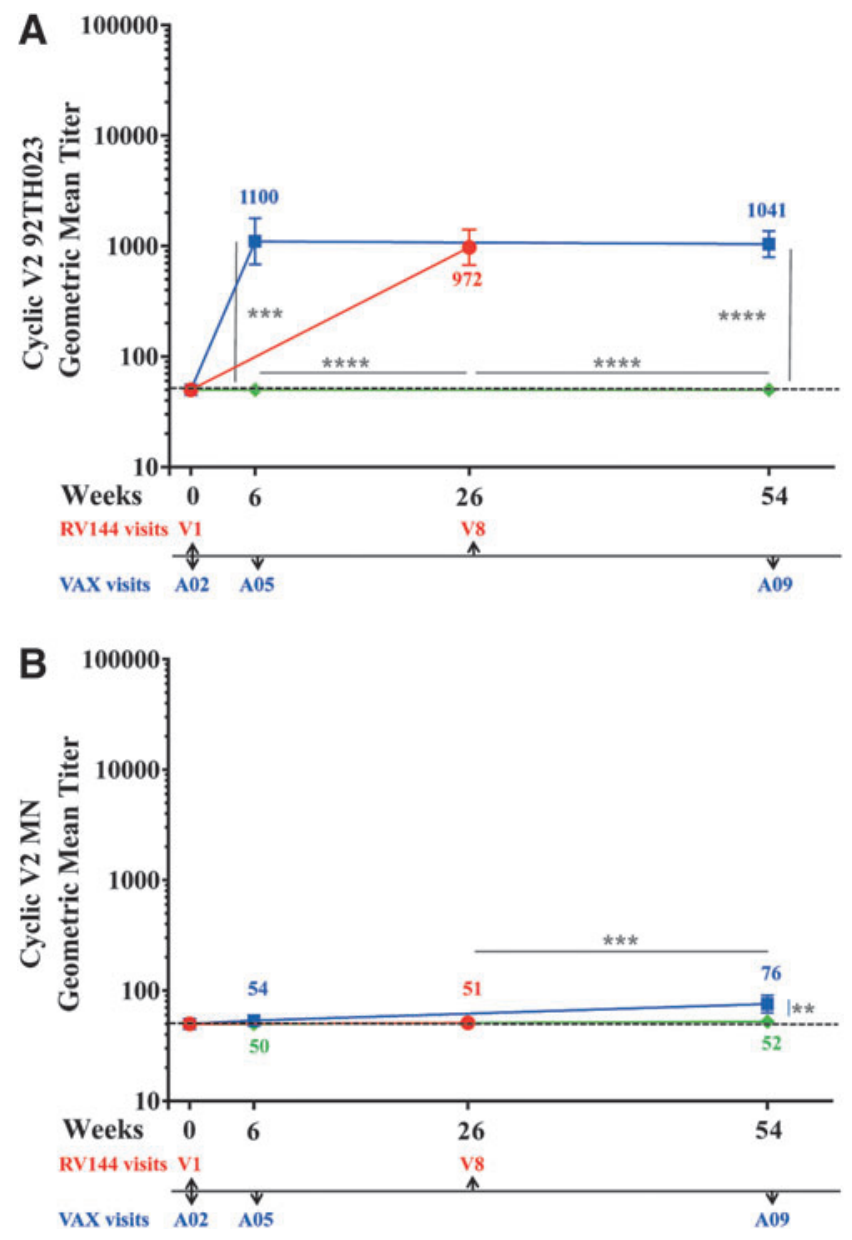

FIG. 5. IgG antibody responses to Cyclic V2 in RV144 and VAX trials. RV144 (red symbols) visits 1 and 8, VAX003 (blue symbols), and VAX004 (green symbols) visits A02, A05, and A09. Color coded numbers are the actual GMTs and symbols are GMTs of IgG binding to (A) cyclic V2 $92 \mathrm{TH} 023$ and (B) cyclic V2 MN with $95 \%$ confidence intervals. Responses above dotted line are considered positive. $* * p<.01 ; * * * p<.001 ; * * * * p<.0001$.

We investigated the durability of V2-specific antibodies using the CycV2 $92 \mathrm{TH} 023$ peptide. In all regimens the antibody responses declined to baseline between vaccinations and multiple boosts did not maintain antibody levels above baseline (data not shown).

\section{IgG antibody subclasses (IgG1-IgG4) to A244gD gp120 envelope}

To characterize IgG subclass responses, we used gp120 $\mathrm{A} 244 \mathrm{gD}$ and samples 2 weeks post last injection in RV144 (visit 8, week 26) and 2 weeks post fourth injection in VAX003 and VAX004 (week 54, A09) (Fig. 6A). All three vaccine regimens induced gp120 IgG1 antibodies with significantly higher responses in VAX003 $(2,082,100 \%)$ followed by RV144 (734, 97\%) and VAX004 (429, 100\%) (Fig. 6A). Low IgG2 responses $(87,52 \%)$ were detected only in VAX003. IgG3 antibodies were detected in VAX003 (299, 94\%) and RV144 (391, 88\%) (Not significant), while IgG3 responses in VAX004 were significantly lower in magnitude and frequency $(55,10 \%)$ than in RV144 and VAX003. IgG4 responses were significantly higher in VAX003 (641, 90\%) than in VAX004 (182, 67\%) and RV144 (51, 3\%) (Fig. 6A).

To determine whether repeated immunizations increase the magnitude of IgG3 antibodies in VAX003 we tested antibody responses to $\mathrm{A} 244 \mathrm{gD}$ measured by absorbance 2 weeks post second, fourth, and fifth protein boosts (Fig. 6B). In VAX003, repeated protein boosts did not increase the magnitude of the $\mathrm{IgG} 3$ responses, with highest level detected 2 weeks post second protein boost (week 6, A05) and lowest 2 weeks post fifth immunization (Fig. 6B). RV144 IgG3 responses were comparable to those of VAX003 two weeks post fourth immunization (week 54, A09).

IgG4 antibodies to $\mathrm{A} 244 \mathrm{gD}$ protein were detected in VAX trials after four protein immunizations (A09) but were not detected in RV144 at peak immunogenicity (visit 8). The lack of IgG4 responses in RV144 might be attributed to the ALVAC vaccinations. We therefore, investigated the presence of IgG4 in VAX003 after two protein immunizations (week 6, A05), the same proteins boosts as in RV144. We showed that only $1 / 50$ vaccinees had IgG4 to A244gD in VAX003 at visit A05, comparable to $1 / 32$ in RV144 at visit 8 (data not shown) suggesting that IgG4 were induced after multiple protein immunizations.

To investigate the $\mathrm{gD}$-specific IgG antibody subclasses, we performed ELISAs using the gD peptide as a capturing antigen (Fig. 6C). Samples from RV144 from visit 8 (week 26) and VAX003 and VAX004 from visit A09 (week 54) were tested for $\mathrm{gD}$ binding antibodies. All vaccine regimens induced gD-specific IgG1 antibodies in 100\%, 97\%, and 96\% of samples in VAX004, VAX003, and RV144, respectively (Fig. 6C). gD-specific IgG3 antibodies were detected in RV144 (73\%) followed by VAX003 (42\%) and VAX004 (8\%). IgG2 responses were only detected in $5 \%$ of samples in VAX003 and none of the vaccines induced IgG4 antibodies to the $\mathrm{gD}$ peptide at the visits tested. Preincubation of samples with the $\mathrm{gD}$ peptide before capturing on ELISA plates inhibited $100 \%$ of the binding indicating the specificity of the antibodies to the peptide (Fig. 6C).

\section{IgG subclass binding to gp70 V1 V2 scaffolds}

We then investigated IgG subclass antibody binding to gp70 V1 V2 $92 \mathrm{TH} 023$ and subtype B Case A2 scaffolds in RV144 at visit 8 and in VAX003 and VAX004 at visit A09 (Fig. 7). VAX003 had significantly higher IgG1 responses to gp70 V1 V2 92TH023 and subtype B Case A2 scaffolds (189 and 44 , respectively) compared to $\operatorname{RV} 144(71,14)$ and VAX004 $(13,21)$ (Fig. 7A, B). IgG3 titers to gp70 V1 V2 $92 \mathrm{TH} 023$ in RV144 were significantly higher (38) than in VAX003 (22) and VAX004 (13). IgG3 responses to gp70 V1 V2 subtype B Case A2 were very low and did not differ significantly between regimens at the sample dilutions tested (Fig. 7B). Due to sample limitations we could not test lower dilutions to detect binding.

\section{Comparison of IgG antibody binding to CycV3 92TH023 and MN peptides}

Antibodies to linear V3 peptides inversely correlated with risk of HIV acquisition in RV144 vaccinees who had low levels of Env-specific plasma IgA ${ }^{28}$ and V3 loop-specific antibodies were associated with imposing immune HIV-1 

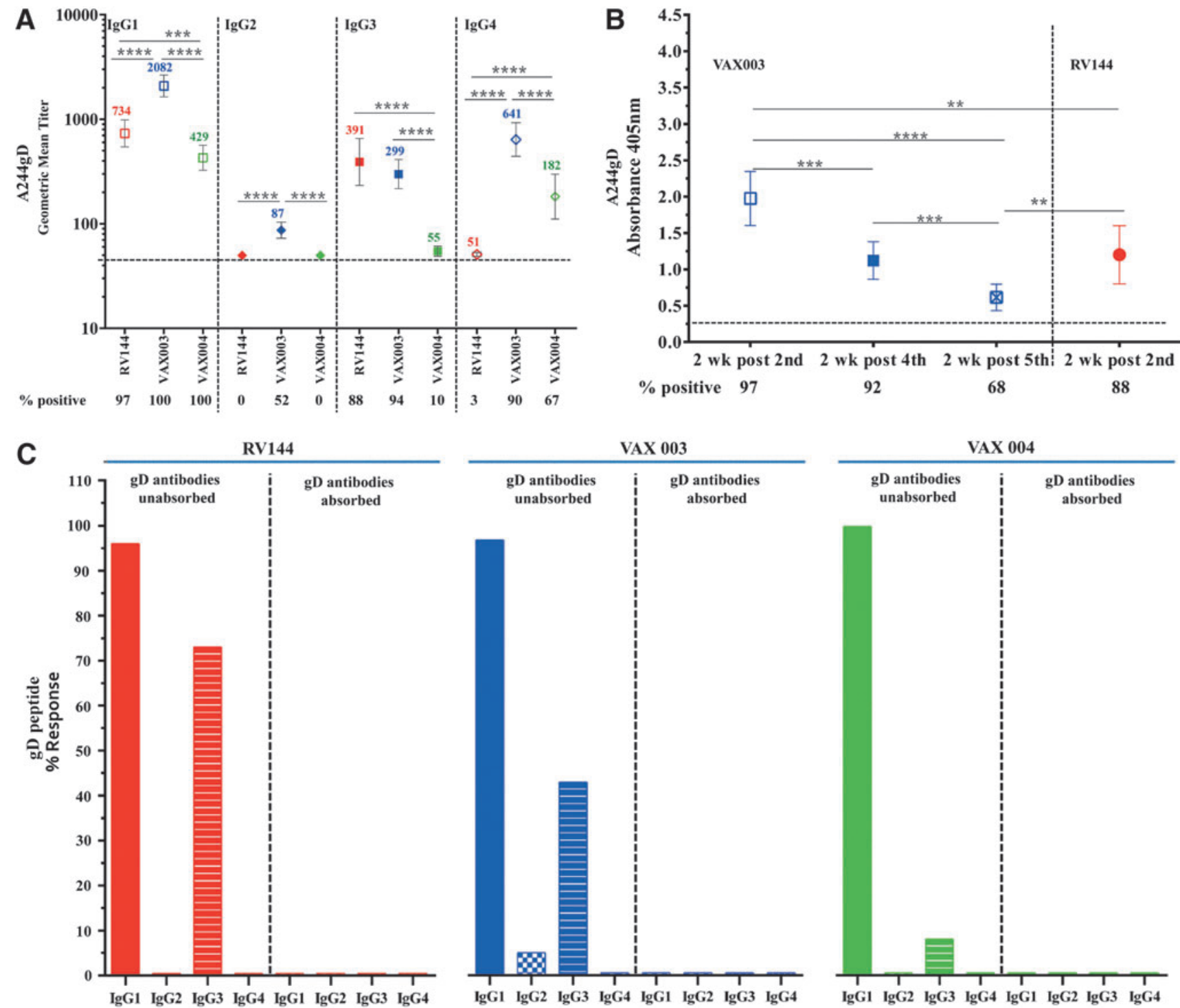

FIG. 6. Comparison of IgG subtypes binding to rgp120 HIV-1 CRF01_AE A244gD protein and gD peptide. (A) IgG subtype (1-4) binding to rgp120 A244gD; RV144 (red symbol) samples at 2-weeks post second (week 26, visit 8), VAX003 (blue symbol) and VAX004 (green symbol) samples at 2-weeks post fourth (week 50, visit A09) vaccination. Color coded numbers in the plot area show GMTs. Numbers under $x$-axis show percentage of positive samples (A405 $\geq 0.25)$ and responses above dotted line are considered positive. Symbols are GMTs with 95\% confidence intervals. *** $p<.001$; $* * * * p<.0001$. (B) IgG3 antibodies binding to rgp120 A244gD; VAX003 (blue symbols) samples at 2-weeks post second (week 6, visit A05), fourth (week 50, visit A09) and fifth (week 74, visit A11), and RV144 at 2-weeks post second (week 26, visit 8) AIDSVAX B/E vaccination. Numbers under $x$-axis show percentage of positive samples (A405 $\geq 0.25$ ) and responses above dotted line are considered positive. Symbols are means and bars indicate standard deviations. $* * p<.01$; $* * * p<.001$; $* * * p<.0001$. (C) Samples were preincubated with HSV-1 gD peptide before adding to ELISA plates with gD peptide as the capturing antigen and compared to gD unabsorbed plasma. Antibody responses with A405 $\geq 0.25$ (2.5 times the background, wells without capturing antigen) were considered positive. Solid colors: IgG1, checkered columns: IgG2, and stripped columns: IgG3. RV144 samples in red at 2-weeks post second injection (visit 8), VAX003 and VAX004 samples, are in blue and green respectively, at 2-weeks post fourth (visit A09) AIDSVAX vaccinations. HSV-1, herpes simplex virus 1 .

infecting viruses pressure on. ${ }^{17}$ Previous studies showed that VAX003 V3-specific antibodies were not significantly different between HIV-infected and uninfected vaccine recipients and did not correlate with the rate of HIV infection. ${ }^{9}$ We therefore, compared the magnitude of antibody responses to $\mathrm{CycV} 3 \mathrm{MN}$ and $\mathrm{CycV} 392 \mathrm{TH} 023$ peptides (Fig. 1B) at baseline (visits 1 and A02, week 0), 2 weeks post second (visit 8, week 26 and A05, week 6) and 2 weeks post fourth protein vaccination in $\mathrm{VAX}$ trials. Antibody responses to both $\mathrm{CycV} 3$ peptides were not detected at baseline in any of the trials (Fig. 8). VAX004 vaccinations did not induce antibodies to $\mathrm{CycV} 392 \mathrm{TH} 023$ and responses remained below cutoff at all visits. After two protein vaccinations response to CycV3 92TH023 were detected in RV144 at visit 8 (104) but 
FIG. 7. IgG1 and $\mathrm{IgG} 3$ antibody responses to HIV-1 gp70 V1 V2 scaffolds in RV144 and VAX samples. Antibody binding to gp70 V1 V2 92TH023 (A) and Case A2 (B) scaffolds in RV144 plasma at 2-weeks post second (visit 8), VAX003 and VAX004 sera at 2-weeks post fourth (visit A09) AIDSVAX B/E vaccination. Hollow symbols show IgG1, solid symbols show IgG3, and color coded numbers show GMT. Numbers below the $x$-axis show percentage of positive samples $(\mathrm{A} 405 \geq 0.25)$ and responses above the dotted line are considered positive. Symbols are GMT with $95 \%$ confidence intervals. $* * p<.01$; $* * * p<.001 ; * * * * p<.0001$.

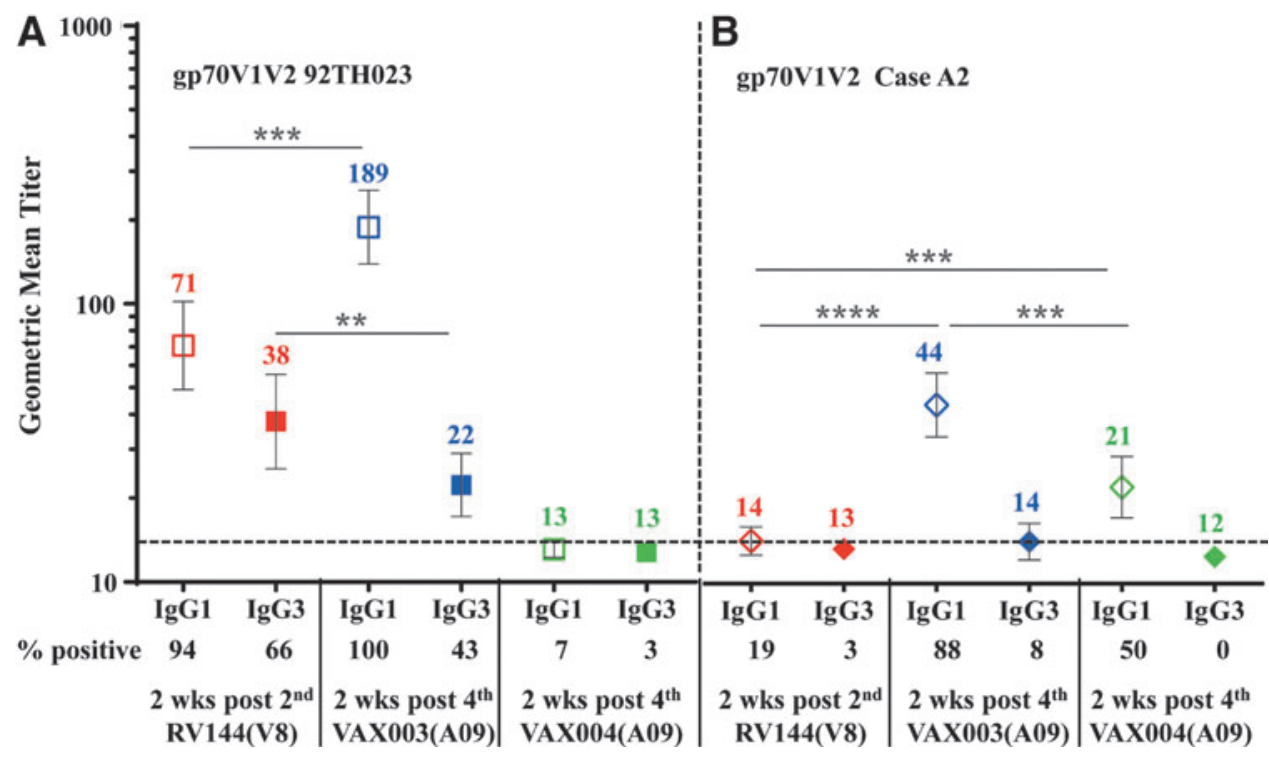

not in VAX003 (A05). Two additional protein boosts in VAX003 (A09) increased the antibody levels (176) above RV144 (Fig. 8).

Responses to $\mathrm{CycV} 3 \mathrm{MN}$ were detected in all trials and were significantly higher than those to CycV3 92TH023.

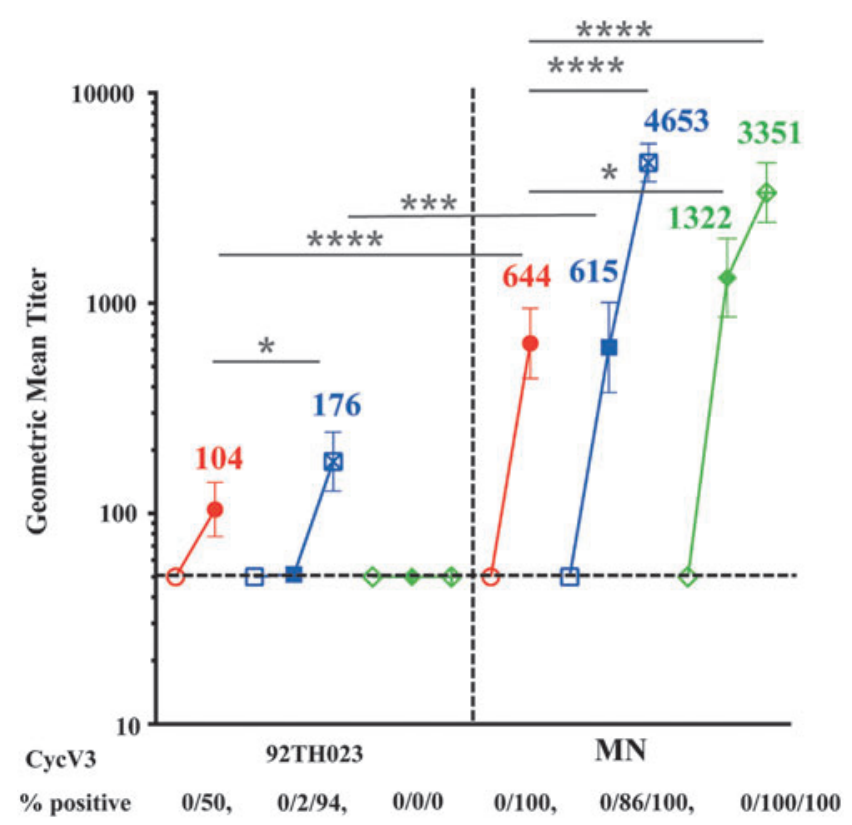

FIG. 8. IgG antibody responses in RV144 and VAX samples to CycV3 $92 \mathrm{TH} 023$ and $\mathrm{MN}$ peptides. CycV3 92 TH023 peptide binding is shown on the left panel and CycV3 MN is on the right. Samples were tested at base line (hollow symbols), 2 weeks post second protein boost (solid symbols) and 2 weeks post forth protein boost (cross symbols). RV144 is in red, VAX003 in blue, and VAX004 in green. Numbers under $x$-axis show percentage of positive samples (A405 $\geq 0.25)$ and responses above dotted line are considered positive. Symbols are GMTs with $95 \%$ confidence intervals and actual GMT values are shown on the panels. $* p<.05 ; * * * p<.001 ; * * * * p<.0001$.
After two protein vaccinations, VAX004 had significantly higher responses $(1,322)$ than in RV144 (644) and VAX003 (615) (Fig. 8). Two additional protein boosts increased the antibody responses in both VAX003 $(4,653)$ and VAX004 $(3,351)$ that were significantly higher than in RV144 at visit 8 .

\section{Characterization of IgA antibody responses to gp120 A244gD in RV144 and VAX trials}

We measured IgA binding to gp120 A244gD in RV144 at peak immunogenicity (visit 8, week 26) and VAX trials at visits $\mathrm{A} 05$ and $\mathrm{A} 09$. In both $\mathrm{VAX}$ trials the frequency and magnitude of the IgA responses at visits A05 and A09 (weeks 6 and 54) were significantly greater than in RV144 at visit 8 (Fig. 9). Multiple protein immunizations in the VAX trials did not increase the magnitude of the IgA response and antibody responses after four protein immunizations (A09) were lower than those after two protein immunizations (A05) (Fig. 9), suggesting that increasing the number of protein boosts does not increase the levels of $\operatorname{IgA}$.

\section{Discussion}

We undertook this analysis to compare multiple facets of the humoral immune response induced by three related HIV vaccine regimens that have been evaluated in efficacy trials. Comparing vaccine responses in relation to behavioral characteristics (heterosexual vs. MSM transmission and PWID), host genetics, antigens, regimens, and viral factors are difficult to determine. We therefore, focused our analysis on comparative relationships limiting speculations on efficacy outcomes.

The number of protein boosts and rest interval appear to influence antibody responses. We showed that the RV144 regimen induced twofold higher antibody titers to one of its cognate vaccine antigens, $92 \mathrm{TH} 023$ Env protein compared to VAX003 after 2 protein boosts but antibody titers to gp120 $\mathrm{MNgD}$ and $\mathrm{A} 244 \mathrm{gD}$ were similar in magnitude (after absorbing the $\mathrm{gD}$ peptide specificity). Neutralization studies using the TZM-bl assay showed that in RV144 neutralization 


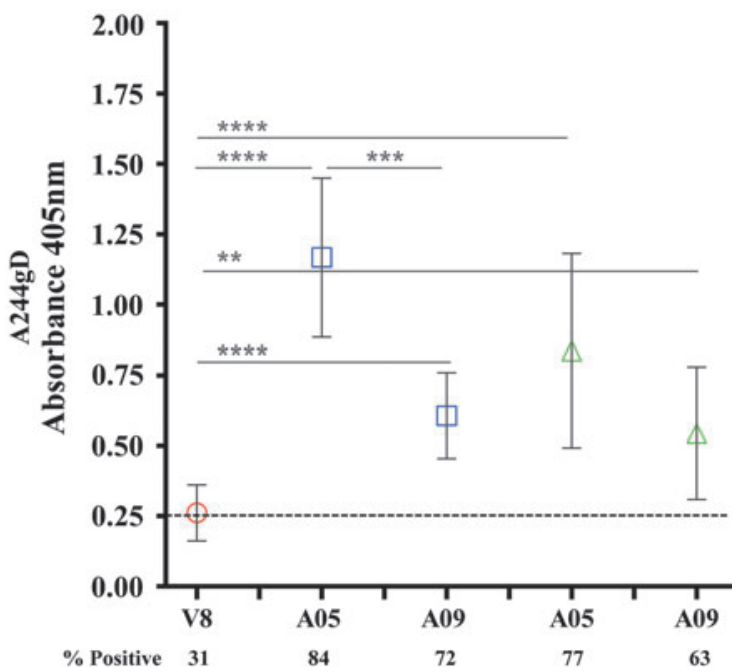

FIG. 9. Comparison of $\operatorname{IgA}$ antibody binding to rgp120 HIV-1 CRF01_AE A244gD in RV144 and VAX samples. Samples were from RV144 (red symbols) at 2 weeks (V8) post second protein boost; VAX003 (blue symbols) at 2 weeks (A05) and post second, and 2 weeks (A09) post fourth protein boost; and VAX004 (green symbols) at 2 weeks (A05) post second, and 2 weeks (A09) post fourth protein boost. Responses above dotted line are considered positive. Symbols are means with $95 \%$ confidence intervals. $* * p<.01 ; * * * p<.001 ; * * * * p<.0001$.

was higher than in VAX003 after two protein injections ${ }^{12}$ suggesting that ALVAC-HIV priming may have contributed to the superior neutralization in RV144. Importantly, in RV144 the two protein vaccinations were given at weeks 12 and 24 and co-administered with ALVAC-HIV, whereas in VAX003 and VAX004 the first two protein vaccinations were given within a month and the short interval between vaccinations may have affected the breath, maturity, and specificity of the initial antibody neutralizing response. In addition, we showed that $\operatorname{IgA}$ antibody responses after two and four protein vaccinations were significantly higher in VAX003 and VAX004 than in RV144. Env specific IgA antibodies from RV144 participants blocked ADCCmediated activity and higher $\mathrm{IgA} / \mathrm{IgG}$ ratios were detected in RV144 infected vaccine recipients. ${ }^{31} \mathrm{We}$ found that repeated protein immunizations in the VAX trials increased the level of antibodies between vaccinations particularly in VAX004 but V1 V2 scaffold and CycV2 antibodies declined significantly between vaccinations in all trials.

In all trials, the $\mathrm{gD}$ sequence was immunogenic and induced IgG and IgA antibodies. We showed that the IgG responses were cross-reactive and bound to both HSV-1 and HSV-2 peptides and pre-existing gD peptide binding antibodies were detected at baseline in both VAX trials but not in the RV144. In VAX003 and VAX004 (visit A05) 50\% of the GMT was attributed to the gD peptide suggesting preexposure of VAX volunteers to HSV and boosting of these responses with the $\mathrm{gD}$ containing AIDSVAX proteins. In RV144, gD responses were relatively low and nonsignificant to the overall gp120 binding. High-risk populations (PWID and MSM), like those enrolled in the VAX trials, are likely to include subjects with high HSV-2 prevalence ${ }^{24,32}$ and therefore higher susceptibility to HIV-1 infection. Induced
gD antibodies failed to prevent HSV-2 infection in the RV144 cohort (Lawrence Corey, pers. comm.). Unfortunately, antibody responses to the gD peptides do not differentiate between HSV-1 and HSV-2 to identify seropositive HSV-2 subjects.

The gD peptide induced $\operatorname{IgG} 1, \operatorname{IgG} 2$, and $\mathrm{IgG} 3$, but not IgG4. Increasing the number of immunizations in the VAX trials decreased the gD peptide-specific responses because IgG4 (not binding to gD) was induced while IgG3 (binding to gD) levels declined. The reasons for IgG4 subclass discrimination to $\mathrm{gD}$ peptide are not clear. IgG4 generation was associated with increased number of protein boosts.

We found that VAX003 immunizations (A09) increased the IgG binding to both scaffolds above RV144 and VAX004. AIDSVAX B/B proteins in VAX004 induced very weak antibody responses to CRF01_AE V1 V2 but responses to subtype B Case A2, after four immunizations, were higher than those in RV144 but significantly lower than in VAX003. Both VAX trials induced high subtype B Case A2 V1 V2 antibodies but lack of efficacy suggests that V1 V2 loop responses alone may not be adequate to provide protection in these groups. Furthermore, the breadth of the V1 V2 loop response could be important for protection. RV144 immunizations induced antibodies to subtypes A, B, C, and CRF01_AE V1 V2 scaffolds. ${ }^{5}$

We showed that responses to $\mathrm{CycV2} \mathrm{MN}$ were poor and detected only in VAX003. RV144 and VAX003 had similar responses to $\mathrm{CycV} 292 \mathrm{TH} 023$ and additional protein boosts in VAX003 did not increase the GMT. CycV2 responses declined in VAX003 with additional immunizations and decline of V1 V2 antibodies could be due to the decline of V2 loop-specific antibodies. In VAX004, CycV2 peptide antibodies were not detected indicating that the binding to subtype B Case A2 V1 V2 scaffold was not in the V2 loop region. V1 V2 scaffolds contain constant region 1 (C1a and $\mathrm{C} 1 \mathrm{~b})$ of gp120 and antibodies were shown before to bind to those sequences. $^{28}$ Importantly, antibody levels to both scaffolds declined to background levels 6 months post vaccinations in all trials. Notably, in HIV-1 infected individual responses to V2 loop are not prominent. ${ }^{14}$

While IgG responses to $\mathrm{CycV} 392 \mathrm{TH} 023$ were detected in RV144 after two protein boosts (visit 8), in VAX003 were not (A05) and antibodies were detected after the fourth protein immunization (A09) indicating that the ALVAC priming in RV144 may have contributed to the earlier generation of V3specific antibodies. After the fourth boost, CycV3 $92 \mathrm{TH} 023$ antibodies in VAX003 increased above those in RV144 while in VAX004 were undetectable.

CycV3 MN antibodies were highest in VAX004 after two protein immunizations indicating that gp120 GNE8 in VAX004 regimen contributed to the generation of subtype B V3 antibodies. Two additional immunizations in the VAX trials increased the antibody titers to MN CycV3 significantly and were higher than in RV144.

Others have shown that RV144 CycV3-specific antibodies were correlated with immune pressure on infecting viruses ${ }^{17}$; however, V3 loop studies were not included in the CoR analysis. Antibodies to linear V3 CRF01_AE peptides were inversely correlated with risk of HIV infection in vaccine recipients with low levels of Env-specific plasma $\operatorname{IgA} .^{28}$ Notably, a substantial fraction of RV144 vaccine-elicited neutralizing Abs to Tier-1 viruses as measured in TZM-bl cells mapped to linear epitopes in the crown of the V3 loop. ${ }^{12}$ 
Our analysis of IgG subclasses showed fundamental differences between the three trials. While VAX003 induced all subclasses of IgG antibodies, RV144 induced IgG1 and IgG3 and VAX004 IgG1 and IgG4. In all vaccines, the predominant IgG subclass was IgG1 and VAX003 (A09) had significantly higher antibody titers than RV144 and VAX004 to all antigens tested. RV144 had higher IgG1 to A244gD and 92TH023 V1 V2 scaffold than VAX004 but VAX004 had significantly higher IgG1 to subtype B Case A2 scaffold indicating that AIDSVAX B/B can induce antibodies to subtype B V1 V2 but not well to CRF01_AE. IgG3 were poorly induced in VAX004 to all antigens tested and were significantly lower than the other two trials. RV144 and VAX003 had similar IgG3 responses to A244gD but RV144 had significantly higher levels of IgG3 to $92 \mathrm{TH} 023 \mathrm{~V} 1 \mathrm{~V} 2$ scaffold. IgG3 binding to the subtype B Case A2 V1 V2 scaffold was weak for both RV144 and VAX003 and undetectable in VAX004. Previous studies showed RV144 and VAX003 to have similar magnitude of responses to subtype B Case A2 scaffold but the rate of responses were significantly higher in RV144 and inversely correlated with risk. ${ }^{15}$ Differences in binding may be explained by the methodologies used (Custom multiplex antibody system, Luminex ${ }^{\circledR}$ vs. ELISA). IgG3 responses to V1 V2 scaffolds are likely to be induced by A244gD since both VAX trials contained MNgD and IgG3 were not detected in VAX004. Since IgG3 antibodies are polyfunctional, this may have affected downstream events with FC receptors. IgG3 antibodies can fix complement, have high affinity for Fc $\gamma$ R:IgG3 $>\operatorname{IgG} 1>\operatorname{IgG} 2>\operatorname{IgG} 4$ (reviewed in Forthal et $a .^{33}$ ), and have been associated with protection in several other infections. ${ }^{34,35}$ Spontaneous control of HIV infection in the absence of antiretroviral therapy is associated with the generation of high levels of p24 and gp120-specific IgG1 and the maintenance of gp120-specific IgG3 antibodies. $^{36}$

We showed that IgG4 antibodies to A244gD were induced in both VAX003 and VAX004 after four protein boosts but not in RV144 after two protein immunizations, agreeing with similar studies. ${ }^{15}$ However, when we investigated the generation of IgG4 in VAX003 after two protein vaccinations we did not detect significant differences between RV144 and VAX003 indicating that more than two protein immunizations are needed to induce IgG4 antibodies. In other studies, multiple HIV-1 Env protein immunizations induced IgG4. ${ }^{37}$ While IgG4 was induced with repeated vaccinations in VAX003, IgG3 decreased gradually and after five protein immunizations responses were significantly lower than earlier boosts and RV144 and just above the background. These observations show a dynamic change of IgG subclass contribution, IgG3 decreasing and IgG4 increasing, to the total IgG with multiple protein immunizations and consequently an alteration of downstream cellular events that depend on the interaction of IgG subclasses with the Fc receptors on effector cells. Therefore, multiple protein boosts can alter the concentration and subclasses of IgG to V1 V2 scaffolds and other epitopes.

Comparison of antibody functionality showed that RV144 induced highly functional IgG3, whereas VAX003 after seven protein immunizations elicited mono-functional antibodies with high levels of IgG4. ${ }^{38}$ IgG subclass switching from highly functional antibodies to IgG4 may pose a new challenge for HIV vaccines as multiple immunizations and vaccine regimens may generate durable antibody responses but antibodies may not be of the highly functional IgG1 and IgG3 subclasses. Factors that determine subclass contribution to total IgG are not fully understood but adjuvants, antigens, vaccine dose, route of administration, age, and vaccine production methodologies could play a role. ${ }^{39}$ Decreased IgG3 and increased of IgG4 and IgG1 subclasses were observed in RV305, a follow-up study in which RV144 uninfected volunteers who received all vaccinations 6-8 years earlier were boosted with ALVAC-HIV/AIDSVAX B/E or AIDSVAX $\mathrm{B} / \mathrm{E}$ alone or ALVAC-HIV alone. ${ }^{40,41}$

In all three HIV-1 trials, the antibody responses observed a typical saw-tooth pattern and were poorly sustained over time. Conflicting results have been observed ${ }^{42,43}$ but none match the long duration of antibody responses observed with other common viral and vaccine antigens. ${ }^{44}$ It is hypothesized that plasma cells are imprinted with a predetermined lifespan based on the magnitude of B cell signaling that occurs during the induction of an antigen-specific humoral immune response. ${ }^{45}$ Moreover, additional AIDSVAX B/E, two late boosts given 8 years post last RV144 vaccination of the ALVAC-HIV/AIDSVAX B/E combination or AIDSVAX B/E alone (RV305) did increase IgG1 and IgG4 but not IgG3 subclass antibodies. ${ }^{40,41}$ However, polyfunctionality in RV144 was tethered to IgG1 and IgG3 responses to Env with IgG3 acting as a surrogate to a coordinated IgG1 and IgG3 response. ${ }^{18}$ Evidence suggests that polyfunctional $\mathrm{CD}^{+} \mathrm{T}$ cells provided help for antibody generation as a correlate in primary analysis. ${ }^{\text {P }}$

\section{Conclusions}

We identified several notable differences in humoral immune responses elicited by preventive vaccine regimens administered in VAX003, VAX004, and RV144. Levels of $\mathrm{IgG}$, and IgG subclasses, and IgA responses to various Env antigens and $\mathrm{gD}$ peptide differed among the three regimens. Higher Env-specific IgA and IgG4 antibodies and decreased IgG3 in the VAX trials compared to RV144 raise the hypothesis that these differences may have contributed to different vaccine efficacy results. However, drawing definitive conclusions regarding immune response correlations with efficacy outcomes is difficult because of differences under which the trials were conducted.

\section{Acknowledgments}

These studies were supported in part by an Interagency Agreement Y1-AI-2642-12 between U.S. Army Medical Research and Materiel Command (USAMRMC) and the National Institutes of Allergy and Infectious Diseases. In addition, this work was supported by a cooperative agreement (W81XWH-07-2-0067) between the Henry M. Jackson Foundation for the Advancement of Military Medicine and the U.S. Department of Defense. This research was funded in part by the U.S. National Institute of Allergy and Infectious Diseases. The authors thank Dr. Marc Gurwith, GSID, for providing serum specimens from VAX003 and VAX004 studies. Ministry of Public Health: Supamit Chunsutthiwat, Vichai Satimai, Prayura Kunasol, Nakorn Premsri, Chawetsan Namwat, Prasert Thongcharoen, Chief Provincial Health Offices of Rayong field sites (Si Racha District, Bang Lamung District, Phan Thong District, Sattahip District) and 
Chon Buri field sites (Muang District, Ban Chang District, Klaeng District, Ban Khai District). Royal Thai Army: Chirapa Eamsila. Military HIV Research Program: Viseth Ngauy, Robert M. Paris, Charla Andrews.

Preliminary results of this study were presented at AIDS Vaccine 2012, September 9-12, 2012, Boston, MA (P03.5LB), and AIDS Vaccine 2013, October 7-10, 2013, Barcelona, Spain (P03.27).

\section{Disclaimer}

The opinions herein are those of the authors and should not be construed as official or representing the views of Mahidol University, the U.S. Department of Health and Human Services, National Institute of Allergy and Infectious Diseases, the Department of Defense, or the Department of the Army.

\section{Author Disclosure Statement}

Co-author James Tartaglia is an employee of Sanofi Pasteur. Co-authors Faruk Sinangil and Donald P. Francis are employees of GSID. Other co-authors declare no competing interests.

\section{References}

1. Rerks-Ngarm S, Pitisuttithum P, Nitayaphan S, et al: Vaccination with ALVAC and AIDSVAX to prevent HIV1 infection in Thailand. N Engl J Med 2009;361:22092220.

2. Robb ML, Rerks-Ngarm S, Nitayaphan S, et al.: Risk behaviour and time as covariates for efficacy of the HIV vaccine regimen ALVAC-HIV (vCP1521) and AIDSVAX B/E: A post-hoc analysis of the Thai phase 3 efficacy trial RV 144. Lancet Infect Dis 2012;12:531-537.

3. Haynes BF, Gilbert PB, McElrath MJ, et al.: Immunecorrelates analysis of an HIV-1 vaccine efficacy trial. N Engl J Med 2012;366:1275-1286.

4. Kim JH, Excler JL, Michael NL: Lessons from the RV144 Thai phase III HIV-1 vaccine trial and the search for correlates of protection. Annu Rev Med 2015;66:423-437.

5. Zolla-Pazner S, Decamp A, Gilbert PB, et al.: Vaccineinduced IgG antibodies to V1 V2 regions of multiple HIV-1 subtypes correlate with decreased risk of HIV-1 infection. PLoS One 2014;9:e87572.

6. Rolland M, Edlefsen PT, Larsen BB, et al.: Increased HIV1 vaccine efficacy against viruses with genetic signatures in Env V2. Nature 2012;490:417-420.

7. Lin L, Finak G, Ushey K, et al.: COMPASS identifies Tcell subsets correlated with clinical outcomes. Nat Biotech 2015;33:610-616.

8. de Souza MS, Ratto-Kim S, Chuenarom W, et al.: The Thai phase III Trial (RV144) vaccine regimen induces T cell responses that preferentially target epitopes within the V2 region of HIV-1 envelope. J Immunol 2012;188:5166-5176.

9. Pitisuttithum P, Gilbert P, Gurwith $\mathrm{M}$, et al.: Randomized, double-blind, placebo-controlled efficacy trial of a bivalent recombinant glycoprotein $120 \mathrm{HIV}-1$ vaccine among injection drug users in Bangkok, Thailand. J Infect Dis 2006; 194:1661-1671.

10. Flynn NM, Forthal DN, Harro CD, Judson FN, Mayer KH, Para MF: Placebo-controlled phase 3 trial of a recombinant glycoprotein 120 vaccine to prevent HIV-1 infection. J Infect Dis 2005;191:654-665.
11. Francis DP, Gregory $\mathrm{T}$, McElrath MJ, et al:: Advancing AIDSVAX to phase 3. Safety, immunogenicity, and plans for phase 3. AIDS Res Hum Retroviruses 1998;14 Suppl 3: S325-S331.

12. Montefiori DC, Karnasuta C, Huang Y, et al:: Magnitude and breadth of the neutralizing antibody response in the RV144 and Vax003 HIV-1 vaccine efficacy trials. J Infect Dis 2012;206:431-441.

13. Gilbert P, Wang M, Wrin T, et al.: Magnitude and breadth of a nonprotective neutralizing antibody response in an efficacy trial of a candidate HIV-1 gp120 vaccine. J Infect Dis 2010;202:595-605.

14. Karasavvas N, Billings E, Rao M, et al.: The Thai phase III HIV type 1 vaccine trial (RV144) regimen induces antibodies that target conserved regions within the V2 loop of gp120. AIDS Res Hum Retroviruses 2012;28:1444-1457.

15. Yates NL, Liao H-X, Fong Y, et al.: Vaccine-induced Env V1-V2 IgG3 correlates with lower HIV-1 infection risk and declines soon after vaccination. Sci Transl Med 2014;6: 228 ra239.

16. Zolla-Pazner S, deCamp AC, Cardozo T, et al:: Analysis of $\mathrm{V} 2$ antibody responses induced in vaccinees in the ALVAC/AIDSVAX HIV-1 vaccine efficacy trial. PLoS One 2013;8:e53629.

17. Zolla-Pazner S, Edlefsen PT, Rolland M, et al:: Vaccineinduced human antibodies specific for the third variable region of HIV-1 gp120 impose immune pressure on infecting viruses. EBioMedicine 2014;1:37-45.

18. Chung AW, Kumar MP, Arnold KB, et al.: Dissecting polyclonal vaccine-induced humoral immunity against HIV using systems serology. Cell 2015;163:988-998.

19. Berman PW: Development of bivalent rgp120 vaccines to prevent HIV type 1 infection. AIDS Res Hum Retroviruses 1998;14 Suppl 3:S277-S289.

20. Lasky LA, Groopman JE, Fennie CW, et al.: Neutralization of the AIDS retrovirus by antibodies to a recombinant envelope glycoprotein. Science 1986;233:209-212.

21. Kayman SC, Wu Z, Revesz K, Chen H, Kopelman R, Pinter A: Presentation of native epitopes in the V1/V2 and V3 regions of human immunodeficiency virus type 1 gp120 by fusion glycoproteins containing isolated gp120 domains. J Virol 1994;68:400-410.

22. Pinter A, Honnen WJ, Kayman SC, Trochev O, Wu Z: Potent neutralization of primary HIV-1 isolates by antibodies directed against epitopes present in the V1/V2 domain of HIV-1 gp120. Vaccine 1998;16:1803-1811.

23. Weiss H: Epidemiology of herpes simplex virus type 2 infection in the developing world. Herpes 2004;11 Suppl 1: $24 \mathrm{~A}-35 \mathrm{~A}$.

24. Holtz TH, Thienkrua W, McNicholl JM, et al.: Prevalence of Treponema pallidum seropositivity and herpes simplex virus type 2 infection in a cohort of men who have sex with men, Bangkok, Thailand, 2006-2010. Int J STD AIDS 2012; 23:424-428.

25. Corey L, Wald A, Celum CL, Quinn TC: The effects of herpes simplex virus- 2 on HIV-1 acquisition and transmission: A review of two overlapping epidemics. J Acquir Immune Defic Syndr 2004;35:435-445.

26. Gupta R, Warren T, Wald A: Genital herpes. Lancet 2007;370:2127-2137.

27. Biraro S, Kamali A, White R, et al.: Effect of HSV-2 on population-level trends in HIV incidence in Uganda between 1990 and 2007. Trop Med Int Health 2013;18:12571266. 
28. Gottardo R, Bailer RT, Korber BT, et al: Plasma IgG to linear epitopes in the V2 and V3 regions of HIV-1 gp120 correlate with a reduced risk of infection in the RV144 vaccine efficacy trial. PLoS One 2013;8:e75665.

29. Peachman KK, Karasavvas N, Chenine A-L, et al:: Identification of new regions in HIV-1 gp120 variable 2 and 3 loops that bind to $\mathrm{a} 4 \mathrm{~b} 7$ integrin receptor. PLoS One 2015; 10:e0143895.

30. O'Connell RJ, Kim JH, Excler JL: The HIV-1 gp120 V1 V2 loop: Structure, function and importance for vaccine development. Expert Rev Vaccines 2014;13:1489-1500.

31. Tomaras GD, Ferrari G, Shen X, et al.: Vaccine-induced plasma IgA specific for the $\mathrm{C} 1$ region of the HIV-1 envelope blocks binding and effector function of IgG. Proc Natl Acad Sci U S A 2013;110:9019-9024.

32. Go VF, Frangakis C, Nam le V, et al:: High HIV sexual risk behaviors and sexually transmitted disease prevalence among injection drug users in Northern Vietnam: Implications for a generalized HIV epidemic. J Acquir Immune Defic Syndr 2006;42:108-115.

33. Forthal D, Hope TJ, Alter G: New paradigms for functional HIV-specific nonneutralizing antibodies. Curr Opin HIV AIDS 2013;8:393-401.

34. Roussilhon C, Oeuvray C, Muller-Graf C, et al.: Long-term clinical protection from falciparum malaria is strongly associated with $\mathrm{IgG} 3$ antibodies to merozoite surface protein 3. PLoS Med 2007;4:e320.

35. Kam YW, Simarmata D, Chow A, et al.: Early appearance of neutralizing immunoglobulin G3 antibodies is associated with chikungunya virus clearance and long-term clinical protection. J Infect Dis 2012;205:1147-1154.

36. Banerjee K, Klasse PJ, Sanders RW, et al.: IgG subclass profiles in infected HIV type 1 controllers and chronic progressors and in uninfected recipients of Env vaccines. AIDS Res Hum Retroviruses 2010;26:445-458.

37. Gorse GJ, Corey L, Patel GB, et al.: HIV-1MN recombinant glycoprotein 160 vaccine-induced cellular and humoral immunity boosted by HIV-1MN recombinant glycoprotein 120 vaccine. National Institute of Allergy and
Infectious Diseases AIDS Vaccine Evaluation Group. AIDS Res Hum Retroviruses 1999;15:115-132.

38. Chung AW, Ghebremichael M, Robinson $\mathrm{H}$, et al.: Polyfunctional Fc-effector profiles mediated by IgG subclass selection distinguish RV144 and VAX003 vaccines. Sci Transl Med 2014;6:228ra238.

39. Aalberse RC, van der Gaag R, van Leeuwen J: Serologic aspects of IgG4 antibodies. I. Prolonged immunization results in an IgG4-restricted response. J Immunol 1983;130: 722-726.

40. Akapirat S, Vasan S, Pitisuttithum P, et al.: Specific IgG subclasses induced in RV305: A late boost vaccination of RV144 subjects. In: The Annual Conference on Retroviruses and Opportunistic Infections (CROI). Boston, MA; Abstract 318; 2016.

41. Williams L, Yates NL, Lucas J, et al.: IgG3 subclass and specificity profiles elicited by the RV305 vaccine regimen. Keystone: HIV Vaccines. Banff, Alberta, Canada, Abstract 5-2016;2015.

42. Goepfert PA, Tomaras GD, Horton H, et al: : Durable HIV1 antibody and T-cell responses elicited by an adjuvanted multi-protein recombinant vaccine in uninfected human volunteers. Vaccine 2007;25:510-518.

43. Excler JL, Tomaras GD, Russell ND: Novel directions in HIV-1 vaccines revealed from clinical trials. Curr Opin HIV AIDS 2013;8:421-431.

44. Amanna IJ, Carlson NE, Slifka MK: Duration of humoral immunity to common viral and vaccine antigens. N Engl J Med 2007;357:1903-1915.

45. Amanna IJ, Slifka MK: Mechanisms that determine plasma cell lifespan and the duration of humoral immunity. Immunol Rev 2010;236:125-138.

Address correspondence to: Nicos Karasavvas Viral Diseases Branch Walter Reed Army Institute of Research Silver Spring, MD 20910

E-mail: nicos.karasavva.mil@mail.mil 